\title{
Long-term Outcomes of Carotid Artery Stenting for Radiation-Associated Stenosis
}

\author{
Min-Ping Huang ${ }^{1}$, Hsiu-Yu Fang ${ }^{1}$, Chung-Yu Chen², Teng-Yeow Tan ${ }^{3}$, Yeh-Lin Kuo ${ }^{4}$, I-Chang Hsieh ${ }^{5}$, \\ Hon-Kan Yip ${ }^{1}$, Chiung-Jen $\mathrm{Wu}^{1}$
}

Background: In Taiwan, the prevalence of head and neck cancer is relatively high. Because radiation-associated carotid stenosis is a significant risk factor for stroke, carotid artery stenting (CAS), instead of carotid endarterectomy, is indicated in patients with radiation-associated carotid stenosis. We sought to evaluate the effect of neck radiotherapy (XRT) on the long-term outcome of patients undergoing CAS.

Methods: $\quad$ From March 2001 to November 2011, 147 CAS procedures were performed on 129 patients ( $n=43$ for XRT, $n=86$ for non-XRT). Mean follow-up was $42.7 \pm 20.5$ months (median: 52 months; range: 1-60 months). Duplex velocity criterion for $>50 \%$ restenosis after CAS was defined as peak systolic velocity $>175 \mathrm{~cm} / \mathrm{s}$. Endpoints included 5-year freedom from mortality, ipsilateral recurrent stroke, and major adverse cardiovascular events (MACE).

Results: $\quad$ The mean age of XRT patients was significantly lesser than that of non-XRT patients $(61 \pm 8$ vs. $71 \pm 8, p<0.001)$. There was significantly less coronary artery disease and other

\begin{abstract}
At a Glance Commentary
Scientific background of the subject

For the higher prevalence of head and neck cancer in Taiwan compared with the West, the long term outcomes of carotid artery stenting between radiation induced and atheroscleroticassociated carotid artery stenosis should be investigated.
\end{abstract}

\section{What this study adds to the field}

We provided the data in Asia population. In the radiation induced carotid artery stenosis group, the mean age was significantly lesser, and greater asymptomatic restenosis rate after stenting was noted. cardiovascular co-morbidities in XRT patients. No significant differences were noted in the composite 30-day ipsilateral stroke/myocardial infarction/mortality (XRT: $8.6 \%$ vs. non-XRT: 6\%, $p>0.05$ ) and 5 -year freedom from mortality, ipsilateral recurrent stroke, and MACE $(p>0.05)$ between the two groups. Intra-stent carotid restenosis $>50 \%$ was significantly higher in the XRT group on follow-up.

Conclusion: Long-term outcomes of CAS for radiation-associated stenosis were not altered by a history of neck XRT, except for asymptomatic carotid restenosis.

(Biomed J 2013;36:144-149)

Key words: carotid artery stenosis, carotid stenting, radiotherapy

$\mathrm{C}$ Yerebrovascular disease remains the leading cause of death worldwide. For those who survive, many are disabled. The most effective way to reduce the impact of this public health problem is prevention. Since stroke is a disease with a variety of pathophysiologic mechanisms, efforts have focused on the different mechanisms underlying stroke, with prevention as the primary aim. Among the various stroke mechanisms, extracranial carotid artery (ECCA) occlusive

From the ${ }^{1}$ Department of Internal Medicine, Division of Cardiology, Kaohsiung Chang Gung Memorial Hospital and Chang Gung University College of Medicine, Kaohsiung, Taiwan; ${ }^{2}$ School of Pharmacy, Kaohsiung Medical University, Kaohsiung, Taiwan;

${ }^{3}$ Neurology, Cerebrovascular Disease Section, Kaohsiung Chang Gung Memorial Hospital and Chang Gung University College of Medicine, Kaohsiung, Taiwan; ${ }^{4}$ Department of Radiology, Kaohsiung Chang Gung Memorial Hospital and Chang Gung University College of Medicine, Kaohsiung, Taiwan; ${ }^{5}$ Department of Internal Medicine, Second Section of Cardiology, Chang Gung Memorial Hospital at Linkou, Chang Gung University College of Medicine, Taoyuan, Taiwan

Received: May 22, 2012; Accepted: Jul. 30, 2012

Correspondence to: Dr. Chiung-Jen Wu and Dr. Hon-Kan Yip, Division of Cardiology, Department of Internal Medicine, Chang Gung Memorial Hospital Kaohsiung Medical Center, Chang Gung University College of Medicine, Kaohsiung, Taiwan. 123, Dapi Rd., Niaosong, Kaohsiung 833, Taiwan (R.O.C.). Tel: 886-7-7317123 ext. 2363; Fax: 886-7-7322402; E-mail: han.gung@ msa.hinet.net

DOI: $10.4103 / 2319-4170.113232$ 
disease is one of the more significant causes which can be prevented by medical treatment alone. As shown in our previous study, ${ }^{[1]}$ although the prevalence of ECCA occlusive disease in Taiwanese stroke patients was found to be much lower compared to Caucasians and was not a significant risk factor for stroke in these patients, ECCA occlusive disease is a significant risk factor for stroke in patients with nasopharyngeal cancer (NPC) treated with head and neck radiation..$^{[2]}$

Carotid stenosis secondary to radiation injury to the carotid arteries is a well-known long-term complication of cervical radiotherapy. ${ }^{[3]}$ Head and neck X-ray therapy (XRT) is a standard and/or adjuvant therapy for head and neck malignancies including NPC, oral and oropharyngeal cancers. Since the prevalence of head and neck cancer in Taiwan is high compared with the West, ${ }^{[4,5]}$ radiation-associated carotid stenosis is not uncommon. In lieu of performing standard carotid endarterectomy (CEA), which may increase the risk of operative complications, carotid artery stenting (CAS) has been suggested in these patients as a minimally invasive alternative. ${ }^{[6]}$ The purpose of this retrospective study was to investigate the effect of neck XRT on the long-term outcome of patients undergoing CAS.

\section{METHODS}

The study protocol was approved by the Institutional Review Committee on Human Research of Kaohsiung Chang Gung Memorial Hospital [institutional review board number: 100-0612B] and all the participants had given their informed consents both for the study and publication of their data. The study was a retrospective review of a database of consecutive unselected patients who underwent CAS at Chang Gung Memorial hospital from March 2001 to November 2011.

\section{Preoperative patient characteristics}

One hundred and forty-seven CAS procedures were performed on 129 patients over a 10-year period. Eighteen patients received CAS on both carotid arteries. Mean follow-up was $42.7 \pm 20.5$ months (median: 52 months; range: 1-60 months). The patients were stratified according to whether or not they exhibited a history of neck XRT. There were 43 patients with a history of XRT 5 years prior to CAS (XRT group). Eighty-six patients without radiation exposure were selected as the control group (non-XRT group) from the Chang Gung Memorial Hospital's database. Patients' baseline medical data included age, sex, preoperative symptomatic transient ischemic attack (TIA)/stroke, history of hypertension, diabetes mellitus (DM), dyslipidemia, smoking, betel nut consumption, chronic kidney disease, peripheral arterial occlusion disease, metabolic equivalent (MET) values $<4$, confirmed coronary artery disease by coronary angiography, and heart failure.

\section{Procedure}

Percentage stenosis of the carotid artery was graded using digital subtraction angiography according to the North American Symptomatic Carotid Endarterectomy Trial (NASCET) criteria. ${ }^{[7]}$ All CAS procedures were performed by an experienced interventionalist under local anesthesia without sedation, using a transfemoral or transbrachial/transradial approach and embolic protection devices. A perioperative antiplatelet regimen consisting of aspirin (100 $\mathrm{mg}$ by mouth daily was started at least $72 \mathrm{~h}$ preoperatively and continued indefinitely postoperatively if no bleeding complication occurred), clopidogrel ( $75 \mathrm{mg}$ by mouth daily with a loading dose of $300 \mathrm{mg}$ one day preoperatively and continued for at least 2 months postoperatively), and intraoperative heparinization was used with the goal of achieving an activated clotting time of 250-350 s.

\section{Endpoints}

The perioperative period was defined as within 30 days after CAS. The perioperative complications included hematoma, periprocedural myocardial infarction, pseudoaneurysm, upper gastrointestinal tract bleeding, acute kidney injury, perioperative cerebrovascular events (TIA or any stroke), and death within 30 days after CAS. The primary endpoints were the occurrence within 5 years of all causes of mortality, ipsilateral recurrent stroke, or major adverse cardiovascular events (MACE), defined as cardiovascular death, myocardial infarction, target vessel revascularization, ipsilateral stroke, and all-cause mortality after the perioperative period. The secondary endpoints were patients' follow-up carotid duplex sonographic data (at least 1 year after CAS), where available, assessed for intra-stent restenosis. We defined carotid artery restenosis $>50 \%$ as a peak systolic velocity greater than $175 \mathrm{~cm} / \mathrm{s}$ at the ipsilateral CCA or ICA, as suggested by the retrospectively applied uniform post-stenting criteria. ${ }^{[8]}$

\section{Statistical analysis}

Categorical variables were assessed using Chi-square or Fisher's exact test. Normally distributed continuous variables were compared using two-tailed, unpaired Student's $t$-tests. All values were represented as a mean \pm standard deviation, where applicable. Secondary outcomes were evaluated using Kaplan-Meier life-table analyses and compared using log-rank analyses. A $p<0.05$ was considered statistically significant. The statistical software package used was SPSS version 14.0 for Windows.

\section{RESULTS}

\section{Baseline characteristics}

The mean age of all XRT patients was significantly lesser compared to those of the non-XRT group (61 \pm 8 ver- 
sus $71 \pm 8, p<0.001)$. There were significantly more patients with CAD, hypertension, hyperlipidemia, DM, and chronic kidney disease in the non-XRT group. Significantly more patients consumed betel nuts in the XRT group. In addition, there was significantly more common carotid artery (CCA) and less internal carotid artery (ICA) involvement in the XRT group [Table 1].

Concerning head and neck cancer type, 30 patients were found to have NPC, 10 patients had oropharyngeal cancer, 2 patients had laryngeal carcinoma, and 1 patient had thyroid cancer. Overall, only seven patients received lymph node dissection or laryngectomy, two had tracheostomy, and one patient received contralateral CEA.

\section{Primary and secondary endpoints}

The perioperative complication rates and perioperative cerebrovascular events, including the combined 30-day myocardial infarction, stroke, and mortality rate, did not differ significantly between XRT and non-XRT patients [Table 2]. There was no mortality during the perioperative period, but four patients in the XRT group had ipsilateral TIA/stroke compared to six patients in the non-XRT group. The primary outcomes, including all-cause mortality, any ipsilateral recurrent TIA/stroke, and MACE did not differ significantly between the two groups in the 5 years of follow-up [Figures 1-3].

As the secondary endpoints, 19 patients in the XRT group and 54 patients in non-XRT group had follow-up carot- id duplex sonographic data for the assessment of intra-stent restenosis. There were significantly more patients with carotid restenosis $>50 \%$ in the XRT group [Table 2]. During the follow-up of 2 years after first CAS, one NPC patient underwent re-stenting of the right CCA due to restenosis of $64 \%$. The re-stented right CCA occluded 2 years later.

\section{DISCUSSION}

Our study population consisted of 43 Taiwanese patients with a history of XRT 5 years before CAS in comparison to 86

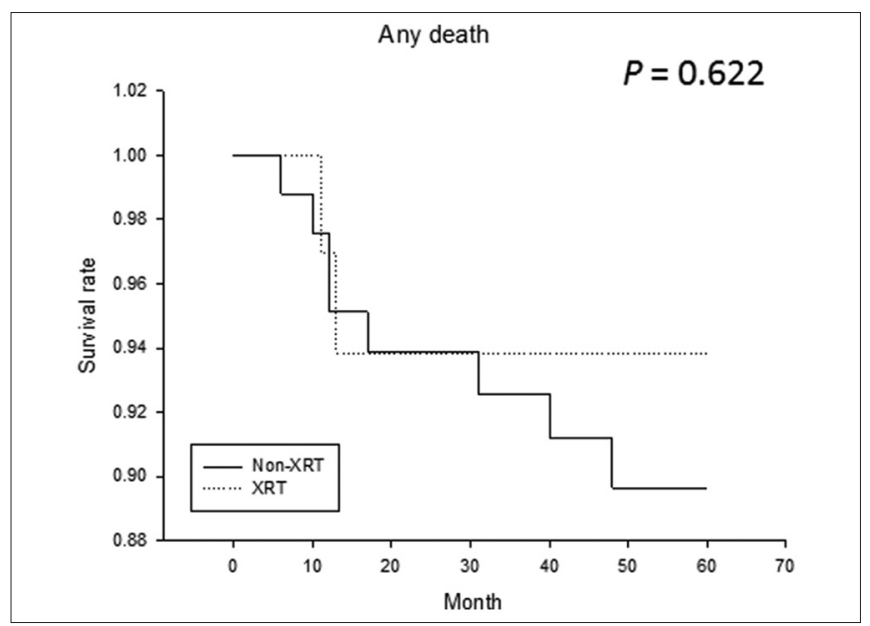

Figure 1: All-cause mortality (XRT, X-ray therapy; non-XRT, non-X-ray therapy)

Table 1: Baseline characteristics of the total population following stratification into XRT and non-XRT patients

\begin{tabular}{|c|c|c|c|}
\hline & XRT $(n=43)$ & Non-XRT $(n=86)$ & $p$ value \\
\hline Age (years) & $61 \pm 8.0$ & $71 \pm 8$ & $<0.0001$ \\
\hline Gender, male & $37(86.0)$ & $72(83.7)$ & 0.731 \\
\hline Symptomatic stroke or TIA & $31(72.1)$ & $50(58.1)$ & 0.122 \\
\hline Coronary artery disease & $9(21.9)$ & $57(66.3)$ & $<0.0001$ \\
\hline Heart failure (NYHA functional class $\geq 2$ ) & $2(4.7)$ & $11(12.8)$ & 0.148 \\
\hline Hypertension & $28(65.1)$ & $76(88.4)$ & 0.002 \\
\hline Diabetes mellitus & $9(20.9)$ & $36(41.9)$ & 0.019 \\
\hline Smoking & $22(51.2)$ & $39(45.3)$ & 0.533 \\
\hline Dyslipidemia & $21(48.8)$ & $61(70.9)$ & 0.014 \\
\hline Peripheral arterial occlusive disease & $0(0)$ & $3(3.5)$ & 0.550 \\
\hline $\mathrm{MET}<4$ & $0(0)$ & $2(2.3)$ & 0.552 \\
\hline Betel nut use & $11(25.6)$ & $1(1.2)$ & $<0.0001$ \\
\hline \multirow[t]{2}{*}{ Chronic renal disease } & $3(7.0)$ & $22(25.6)$ & 0.012 \\
\hline & XRT $(n=47)$ & Non-XRT $(n=100)$ & $p$ value \\
\hline \multicolumn{4}{|l|}{ Characteristics of target vessels } \\
\hline Percent carotid artery stenosis & $77 \pm 11$ & $77 \pm 11$ & 0.798 \\
\hline Internal carotid artery stenosis & $23(48.9)$ & $77(77.0)$ & 0.001 \\
\hline Common carotid artery stenosis & $12(25.5)$ & $10(10.0)$ & 0.014 \\
\hline Both internal and common artery stenosis & $12(25.5)$ & $13(13.0)$ & 0.059 \\
\hline
\end{tabular}

One hundred and forty-seven procedures were performed on 129 patients, Data expressed as mean \pm standard deviation or number (\%), The level of statistical significance was set at $p<0.05$

Abbreviations: XRT: X-ray therapy; non-XRT: Non-X-ray therapy; TIA: Transient ischemic attack; NYHA: New York Heart Association; MET: Metabolic equivalent 
Table 2: Perioperative complications and endpoints

\begin{tabular}{|c|c|c|c|}
\hline & XRT $(n=47)$ & Non-XRT $(n=100)$ & $p$ value \\
\hline \multicolumn{4}{|l|}{ Perioperative complications ( $<30$ days) } \\
\hline Hematoma & $0(0)$ & $2(2.0)$ & 1.000 \\
\hline Periprocedural myocardial infarction & $0(0)$ & $0(0)$ & - \\
\hline Pseudoaneurysm & $0(0)$ & $1(1.0)$ & 1.000 \\
\hline Upper gastrointestinal bleeding & $0(0)$ & $1(1.0)$ & 1.000 \\
\hline Acute renal injury & $0(0)$ & $2(2.0)$ & 1.000 \\
\hline Ipsilateral TIA/stroke & $4(8.6)$ & $6(6.0)$ & 0.287 \\
\hline Mortality & $0(0)$ & $0(0)$ & - \\
\hline \multirow[t]{2}{*}{ Ipsilateral TIA/stroke/mortality } & $4(8.6)$ & $6(6.0)$ & 0.287 \\
\hline & XRT $(n=19)$ & Non-XRT $(n=54)$ & \\
\hline \multirow[t]{2}{*}{ Intra-stent restenosis $>50 \%$ at 36 months } & $3(15.8)$ & $1(1.9)$ & 0.022 \\
\hline & XRT $(n=43)$ & Non-XRT $(n=86)$ & $p$ value \\
\hline \multicolumn{4}{|l|}{ 5-year survival outcome } \\
\hline Long-term all-cause mortality & $2(4.7)$ & $8(9.3)$ & 0.622 \\
\hline Long-term any ipsilateral recurrent TIA/stroke & $5(11.6)$ & $7(8.1)$ & 0.222 \\
\hline Long-term MACE & $7(16.2)$ & $16(18.6)$ & 0.643 \\
\hline
\end{tabular}

One hundred and forty-seven procedures were performed on 129 patients, Data expressed as mean \pm standard deviation or number (\%), The level of statistical significance was set at $p<0.05$

Abbreviations: XRT: X-ray therapy; non-XRT: Non-X-ray therapy; TIA: Transient ischemic attack; MACE: Major adverse cardiovascular events

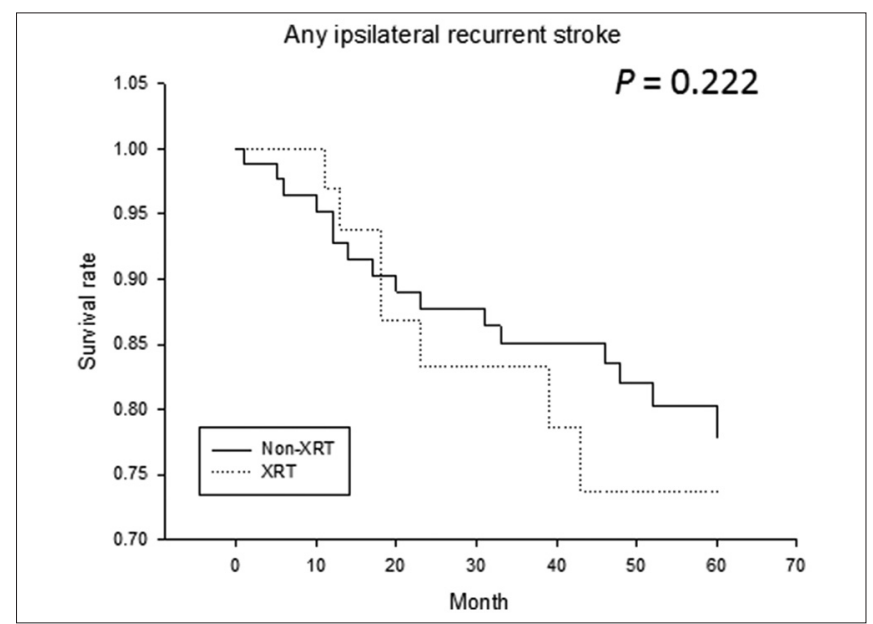

Figure 2: Any ipsilateral recurrent stroke (XRT, X-ray therapy; non-XRT, non-X-ray therapy)

Taiwanese patients who received CAS without XRT exposure. Information regarding such a large number of XRT patients is a unique characteristic of our study when compared to similar studies performed in the West. ${ }^{[9,10]}$ The mean age of our XRT patients was significantly lesser compared to the non-XRT patients. In addition, there were significantly fewer patients with $\mathrm{CAD}$, hypertension, hyperlipidemia, $\mathrm{DM}$, and chronic kidney disease in our XRT group, and this finding differed from similar studies reported in the Western literature. ${ }^{[9,10]}$

The perioperative complications and primary outcomes, all causes of mortality, ipsilateral recurrent stroke, and MACE, did not significantly differ between the XRT and non-XRT patients, as noted previously..$^{[9,10]}$ However, a significantly higher restenosis rate was observed in our XRT



Figure 3: Major adverse cardiovascular events (XRT, X-ray therapy; non-XRT, non-X-ray therapy; MACE, major adverse cardiovascular events)

patients, which correlated with previous studies. ${ }^{[9]}$

Regarding baseline preoperative characteristics and comorbidities between the XRT and non-XRT patients, one prior study showed no difference in age, gender, hypertension, DM, dyslipidemia, smoking, chronic kidney disease, chronic obstructive pulmonary disease, coronary artery disease, and heart failure ${ }^{[9]}$ and another showed differences only with respect to hypertension and gender. ${ }^{[10]}$ This is in contradistinction to our findings where the XRT patients were significantly younger in age compared to the non-XRT patients. The epidemiology of NPC, oral and oropharyngeal cancer is quite different in Taiwan compared with the West, ${ }^{[4,5]}$ and the median age of diagnosis is significantly lower than in the West. The etiology for the 
higher incidence of NPC in Taiwan includes Epstein-Barr virus infection interaction, environmental factors (irritant fumes, certain preserved foods rich in nitroso compounds and volatile nitrosamines), and a genetic component. ${ }^{[4,11]}$ Moreover, the incidence of oral and oropharyngeal cancer rises in Taiwan annually due to increased consumption of betel quid, primarily in young adult males. ${ }^{[12]}$ We believe this is the underlying reason for the significantly younger age of the XRT patients in our study compared with the other studies in the West. ${ }^{[9,10,13]}$

In patients who have undergone neck XRT for malignancy, chronic radiation vasculopathy affects both intra- and extracranial arteries, with increasing rates of stenosis over time. ${ }^{[14]}$ The relative risk of TIA or ischemic stroke is at least doubled, ${ }^{[15]}$ and the interval between the completion of XRT and the stroke event is more than 10 years. ${ }^{[2]}$ Our data showed that the CCA lesions were more frequently affected by XRT in our patients, in accordance with other studies..$^{[9,16]}$ Previous studies have shown that radiation-induced carotid stenosis is more diffuse in distribution and has a different sonographic plaque characterization (less shadowing calcification but higher incidence of hypoechoic plaque) compared with carotid stenosis without radiation exposure, ${ }^{[16]}$ possibly due to instability of the plaque in the XRT patients. ${ }^{[17,18]}$ Radiation dose was found to have no significant influence on the progression of atherosclerosis when a total dose of more than 40 Gy or even higher than 55 Gy was used. ${ }^{[19,20]}$

Significantly higher restenosis rates in XRT patients were observed in our study; however, the restenosis rate was not so high. ${ }^{[9]}$ We know that aging is a major risk factor for atherosclerosis and carotid artery disease. The younger age of our XRT patients may mask the risk of restenosis. Although we found no significant difference in the secondary outcomes between XRT and non-XRT patients in 5 years of follow-up, further data from studies with longer follow-up are needed.

The patients with radiation-associated carotid stenosis were labeled as "high risk" for standard CEA; however, a current review showed that patients treated with CEA had lower rates of late cerebrovascular adverse events and restenosis compared with those treated with CAS. ${ }^{[21]}$ Therefore, the choice for revascularization therapy should be considered on an individual basis.

Our study had several limitations. First, since only a small percentage of patients undergoing CAS had a history of neck XRT, there was a significant discrepancy in the sample sizes between our two groups of patients (XRT vs. non-XRT). Second, our patients were followed at different outpatient departments by different neurologists and/or cardiologists. The rate of routine follow-up carotid duplex examination in this entire cohort was only approximately $80 \%$ in the first year and $70 \%$ by the third year. Another important limitation of our study was the inability to distinguish radiation-induced ICA stenosis from other etiologies such as atherosclerotic occlusive disease. The decreased incidence of coronary artery disease in our XRT patients, however, supported our assumption that all occlusions were radiation induced. Furthermore, because of the retrospective nature of our study design (analysis of the carotid artery stenosis patients with CAS at our hospitals), our study can neither provide data on the prevalence of radiation-induced carotid artery disease nor on the prevalence of stroke in NPC, oral and oropharyngeal cancer patients. Nevertheless, other potential causes of stroke, which could play a role in XRT patients, such as hypercoagulable state, have not been checked. ${ }^{[22]}$ However, a hypercoagulable state was not anticipated since most patients were not in an active malignancy state. Finally, it is difficult to evaluate the effect of the difference in age between XRT and non-XRT patients, which may mask a higher restenosis rate in XRT patients. Physicians should be aware of the long-term complications of XRT in patients and evaluate the extracranial carotid arteries, especially 5 years after the completion of radiotherapy, with or without stroke. ${ }^{[14]}$ In all XRT patients after CAS, routine follow-up carotid duplex examination at 1 month, 6 months, and annually is indicated and is felt to be cost effective. ${ }^{[23]}$

\section{CONCLUSION}

We demonstrated that XRT patients receiving CAS in Taiwan were significantly younger and had more underlying cardiovascular comorbidities, compared to the non-XRT group. Long-term outcomes of CAS for radiation-associated stenosis were not altered by a history of neck XRT, except for asymptomatic carotid restenosis.

\section{REFERENCES}

1. Tan TY, Chang KC, Liou CW, Schminke U. Prevalence of carotid artery stenosis in Taiwanese patients with one ischemic stroke. J Clin Ultrasound 2005;33:1-4.

2. Li CS, Schminke U, Tan TY. Extracranial carotid artery disease in nasopharyngeal carcinoma patients with post-irradiation ischemic stroke. Clin Neurol Neurosurg 2010;112:682-6.

3. Abayomi OK. Neck irradiation, carotid injury and its consequences. Oral Oncol 2004;40:872-8.

4. Chang ET, Adami HO. The enigmatic epidemiology of nasopharyngeal carcinoma. Cancer Epidemiol Biomarkers Prev 2006;15:1765-77.

5. Warnakulasuriya S. Global epidemiology of oral and oropharyngeal cancer. Oral Oncol 2009;45:309-16.

6. Friedell ML, Joseph BP, Cohen MJ, Horowitz JD. Surgery for carotid artery stenosis following neck irradiation. Ann Vasc Surg 2001;15:13-8.

7. Beneficial effect of carotid endarterectomy in symptomatic patients with high-grade carotid stenosis. North American Symptomatic Carotid 
Endarterectomy Trial Collaborators. N Engl J Med 1991;325:445-53.

8. Setacci C, Chisci E, Setacci F, Iacoponi F, de Donato G. Grading carotid intrastent restenosis: A 6-year follow-up study. Stroke 2008;39:1189-96.

9. Protack CD, Bakken AM, Saad WE, Illig KA, Waldman DL, Davies MG. Radiation arteritis: A contraindication to carotid stenting? J Vasc Surg 2007;45:110-7.

10. Sadek M, Cayne NS, Shin HJ, Turnbull IC, Marin ML, Faries PL. Safety and efficacy of carotid angioplasty and stenting for radiation-associated carotid artery stenosis. J Vasc Surg 2009;50:1308-13.

11. Klein G. Nasopharyngeal carcinoma (NPC) is an enigmatic tumor. Semin Cancer Biol 2002;12:415-8.

12. Ho PS, Ko YC, Yang YH, Shieh TY, Tsai CC. The incidence of oropharyngeal cancer in Taiwan: An endemic betel quid chewing area. J Oral Pathol Med 2002;31:213-9.

13. Favre JP, Nourissat A, Duprey A, Nourissat G, Albertini JN, Becquemin JP. Endovascular treatment for carotid artery stenosis after neck irradiation. J Vasc Surg 2008;48:852-8.

14. Cheng SW, Wu LL, Ting AC, Lau H, Lam LK, Wei WI. Irradiation-induced extracranial carotid stenosis in patients with head and neck malignancies. Am J Surg 1999;178:323-8.

15. Plummer C, Henderson RD, O'Sullivan JD, Read SJ. Ischemic stroke and transient ischemic attack after head and neck radiotherapy: A review. Stroke 2011;42:2410-8.

16. Lam WW, Liu KH, Leung SF, Wong KS, So NM, Yuen HY, et al. Sonographic characterisation of radiation-induced carotid artery stenosis. Cerebrovasc Dis 2002;13:168-73.

17. Reilly LM, Lusby RJ, Hughes L, Ferrell LD, Stoney RJ, Ehrenfeld WK. Carotid plaque histology using real-time ultrasonography. Clinical and therapeutic implications. Am J Surg 1983;146:188-93.

18. Langsfeld M, Gray-Weale AC, Lusby RJ. The role of plaque morphology and diameter reduction in the development of new symptoms in asymptomatic carotid arteries. J Vasc Surg 1989;9:548-57.

19. Chung TS, Yousem DM, Lexa FJ, Markiewicz DA. MRI of carotid angiopathy after therapeutic radiation. J Comput Assist Tomogr 1994;18:533-8.

20. Steele SR, Martin MJ, Mullenix PS, Crawford JV, Cuadrado DS, Andersen CA. Focused high-risk population screening for carotid arterial stenosis after radiation therapy for head and neck cancer. Am J Surg 2004;187:594-8.

21. Fokkema M, den Hartog AG, Bots ML, van der Tweel I, Moll FL, de Borst GJ. Stenting versus surgery in patients with carotid stenosis after previous cervical radiation therapy: Systematic review and meta-analysis. Stroke 2011;43:793-801.

22. Cestari DM, Weine DM, Panageas KS, Segal AZ, DeAngelis LM. Stroke in patients with cancer: Incidence and etiology. Neurology 2004;62:2025-30.

23. American College of Cardiology Foundation; American Society of Interventional and Therapeutic Neuroradiology; Society for Cardiovascular Angiography and Interventions; Society for Vascular Medicine and Biology; Society of Interventional Radiology, Bates ER, Babb JD, Casey DE Jr, Cates CU, Duckwiler GR, Feldman TE, et al. ACCF/SCAI/SVMB/SIR/ASITN 2007 clinical expert consensus document on carotid stenting: A report of the American College of Cardiology Foundation Task Force on Clinical Expert Consensus Documents (ACCF/SCAI/SVMB/SIR/ASITN Clinical Expert Consensus Document Committee on Carotid Stenting). J Am Coll Cardiol 2007;49:126-70. 